Original Paper http://ajol.info/index.php/ijbcs http://indexmedicus.afro.who.int

\title{
Politique publique de gestion des espaces verts de la ville de Lomé au Togo
}

\author{
Botolisam POLORIGNI*, Raoufou A. RADJI et Kouami KOKOU \\ Laboratoire de Botanique et Ecologie Végétale, Département de Botanique, Faculté des Sciences, Université \\ de Lomé, BP 1515, Lomé, TOGO. \\ *Auteur correspondant ; E-mail : botolisam@yahoo.fr ; Tel : (228) $90048139 / 22208114$; \\ BP 1515-Lomé 01-Togo
}

\section{RESUME}

Lomé, capitale du Togo, a connu des études qui ont montré que les espaces verts qui étaient inséparables de la ville à l'origine n'ont pas suivi l'extension spatiale et ont même disparu. Ainsi, Lomé est caractérisée par un environnement de plus en plus urbanisé où le besoin de verdure et de fleurs est de plus en plus ressenti. Cela pose donc un problème de gestion de ces espaces verts en l'occurrence un problème de politique publique de gestion des espaces verts. Une prospection de la ville de Lomé a permis de répertorier, quantifier et de dresser la typologie des espaces verts. Des interviews ont permis d'établir le diagnostic du cadre institutionnel et juridique de ces espaces verts. Il ressort de cette étude que chaque habitant de la ville de Lomé dispose de $0,75 \mathrm{~m}^{2} \mathrm{~d}$ 'espace vert contre une norme de $10 \mathrm{~m}^{2}$ préconisée par l'Organisation Mondiale de la Santé. Et pourtant, des cadres institutionnel et juridique de mise en valeur et gestion des espaces verts existent et sont incarnés par des services publics (la direction des espaces verts, des monuments historiques et de l'embellissement ; l'atelier espaces verts de la municipalité et le service technique de la préfecture du Golfe) et la loi n²008-005 du 30 mai 2008 portant loi-cadre sur l'environnement. Cette étude fait un bilan de la politique publique et de l'état des lieux de la gestion des espaces verts et analyse les dysfonctionnements et manquements liés aux cadres institutionnel et juridique.

(C) 2015 International Formulae Group. All rights reserved.

Mots clés : Espaces verts, politique publique, gestion municipale, Lomé.

\section{Public policy of management of green spaces of the city of Lomé in Togo}

\begin{abstract}
Some studies done in Lomé, the capital city of Togo, have shown that the green spaces which at the beginning were inseparable from the city did not follow the space extension and have even disappeared today. Therefore, Lomé is characterized today by an environment that is more and more urbanized and where the need for greenery and the flowers is more felt. This leads to a problem that is the management of these green spaces known as the problem of public of green spaces management policy. A prospecting of the city of Lomé made it possible to index, quantify and to draw up the typology of green spaces. Interviews let to establish the diagnosis of the institutional and legal framework of the green spaces. From this study it comes out that each
\end{abstract}


inhabitant of Lomé only has $0,75 \mathrm{~m}^{2}$ of green spaces against a norm of $10 \mathrm{~m}^{2}$ that is recommended by the World Health Organization. Yet, the executive and law institutions that regularize the management of green spaces exist and are incarnated by public services (management of green spaces, historic monuments and embellishment) and the law n ${ }^{\circ} 2008-005$ of 30 may 2008 concerning the framework law on the environment. This study is an assessment of the public policy and the inventory management of green spaces. It also analyzes the dysfunctioning and failures related to the institutional and legal framework.

(C) 2015 International Formulae Group. All rights reserved.

Keywords : Green spaces, public policies, municipal management, Lomé.

\section{INTRODUCTION}

Dans un monde de plus en plus urbanisé et face aux enjeux du développement durable, l'espace vert apparaît comme indispensable au développement harmonieux de la ville (Ali-Kodja, 2010). Les espaces verts sont les principaux îlots de nature et de surface conséquente dans le tissu urbain (Mehdi et Di Pietro, 2009) et s'inscrivent comme une réponse voire un rempart au mouvement continu d'urbanisation (Choumert, 2009).

Du square au jardin public, les bienfaits des espaces verts par catégorie sont multiples. Ils sont liés à la santé générale et psychologique (Van Dillen et al., 2012) ; à la réduction des symptômes cardiovasculaires et des troubles respiratoires (Richardson et al., 2010); à l'amélioration de la capacité de concentration (Taylor et al., 2009); au renforcement de l'attachement communautaire (Arnberger et Renate, 2012); à la conservation de la biodiversité (Säumel et al., 2010); à la régulation de la température (Loughner et al., 2012) ; à la séquestration du carbone (Davies et al., 2011) ; à la réduction des risques d'inondation (Kubal et al., 2009) et à une plus-value immobilier (Hoshino et Kuriyama, 2010).

Dans la plupart des villes d'Afrique subsaharienne où le développement des villes échappe au contrôle des pouvoirs publics et des planificateurs, les espaces verts sont peu valorisés et sujets à une mauvaise gestion (Kombaté, 2004 ; Dakissaga, 2006 ; Nassa, 2009 ; Ali, 2009 ; Kassay, 2010). Lomé, capitale du Togo, n'est pas en marge de cette situation. Avec 1,5 million d'habitants représentant $63 \%$ des citadins du pays
(DGSCN, 2010), les espaces verts de cette ville apparaissent déficitaires (Polorigni, 2012) et en déphasage avec sa croissance. Les espaces verts prévus par les plans d'urbanisme sont soit non matérialisés sur le terrain du fait de la forte pression foncière soit squattés par les populations au profit des marchés pirates avec le flot de déchets quotidiens (Kassay, 2010) et des maisons d'habitation. Les quelques rares espaces verts aménagés subissent l'assaut des activités culturales. Ils deviennent le paradis des occupants clandestins (Elom, 2011).

La situation des espaces verts de la ville de Lomé semble dénoter d'un désengagement et d'une impuissance des acteurs publics chargés du développement de ce secteur d'activité. Il est donc question dans cette étude de faire un état des lieux des espaces verts de la ville de Lomé et en dresser le bilan de leur gestion à travers une analyse des cadres institutionnel et juridique existants.

\section{MATERIEL ET METHODES \\ Description du milieu d'étude}

La zone d'étude Lomé, est un espace géographique partagé entre deux sous-zones : la commune de Lomé subdivisée en 5 arrondissements et les cantons périphériques (Aflao-Sagbado, Togblékopé, Sanguéra, Agoènyivé, Légbassito et Baguida) (Figure 1). Elle s'étend sur une superficie de $280 \mathrm{~km}^{2}$ (Guezere, 2008), entre $6^{\circ}$ latitude Nord et $1^{\circ} 15$ longitude Est. Située dans la plaine côtière du sud-Togo, Lomé jouit d'un climat de type subéquatorial guinéen caractérisé par deux saisons de pluies (avril à juillet et septembre à octobre) et deux saisons sèches (novembre à mars et août). La pluviosité 
moyenne annuelle est de $800 \mathrm{~mm}$ et la température moyenne est de $29{ }^{\circ} \mathrm{C}$ (Direction de la météorologie nationale, 2012). L'humidité relative est de $57-58 \%$ en saison sèche et $71-73 \%$ en saison pluvieuse. Les sols sont à texture sableuse sur le cordon littoral et ferrugineux sur le plateau de barre (Adjaho, 2010). La flore de la plaine côtière est une mosaïque d'îlots forestiers disparates et de reliques de forêts sacrées (Kokou, 1998), de savanes anthropisées et de prairies inondables, de fourrés littoraux de jachères, de cultures, de plantations (Akpagana et al., 1993 ; Batawila, 1997) et de mangroves (Afidégnon, 1999 ; CC-PNAE, 2003).

\section{Collecte des données}

Une prospection dans la ville de Lomé a permis de réaliser un état des lieux des espaces verts, d'en établir un classement pour l'obtention d'une typologie basée sur le nom de l'aménagement. Les espèces dominantes ont été relevées en présence/absence.

La proportion d'espace vert par habitant de la ville de Lomé a été établie en comparaison avec la norme définie par l'Organisation Mondiale de la Santé (OMS) (Institut d'Aménagement et d'Urbanisme, 2009).

Des entretiens et interviews avec les responsables des services en charge de la gestion des espaces verts ont permis de relever les compétences de chaque service.

Une analyse des cadres institutionnel et juridique a permis d'identifier les dysfonctionnements.

\section{RESULTATS}

\section{Etat des lieux des espaces verts de la ville} de Lomé

Les espaces verts de la ville de Lomé inventoriés sont composés des arbres en alignement, de jardins publics (photo 1, A et
B), de jardins privés (Photo 2) et des aires de jeux.

L'inventaire réalisé (Tableau 1) fait apparaître une répartition hétérogène des types d'espaces verts de la ville de Lomé. On distingue d'une part une concentration de jardins publics et176 km de voies bitumées bordées d'essences arborées dans le quartier administratif contigu à la plage au Sud et limité au Nord par la lagune. Ces arbres sont disposés en des plantations homogènes et discontinues par endroit. D'autre part, l'ensemble des périphéries de la ville, densément bâti, offre peu d'artères pourvues de plantations d'arbres et de jardins publics aménagés en espaces verts.

La superficie totale des espaces verts inventoriés (1 $108828 \mathrm{~m}^{2}$ soit 110,88 hectares) représente $0,40 \%$ de la superficie de l'agglomération de Lomé (28 000 hectares). Le rapport entre cette superficie et la population totale estimée est de 0,75 $\mathrm{m}^{2} /$ habitant. Ce rapport représente la proportion d'espace vert disponible par habitant à Lomé. Ce chiffre est nettement inférieur à la norme de l'Organisation Mondiale de la Santé qui préconise $10 \mathrm{~m}^{2}$ d'espace vert par habitant et exprime ainsi le déficit en espaces verts de la ville capitale du Togo.

\section{Cadre institutionnel et administratif de gestion des espaces verts}

Cette étude a révélé qu'au Togo, trois principaux acteurs interviennent dans la gestion des espaces verts. Ce sont la Direction des Espaces Verts, des Monuments Historiques et de l'Embellissement (DEVMHE) du ministère de l'urbanisme et de l'habitat ; l'atelier espaces verts de la direction des services techniques de la municipalité de Lomé ; et le service technique de la préfecture du Golfe. 
B. POLORIGNI et al. / Int. J. Biol. Chem. Sci. 9(4): 1888-1901, 2015

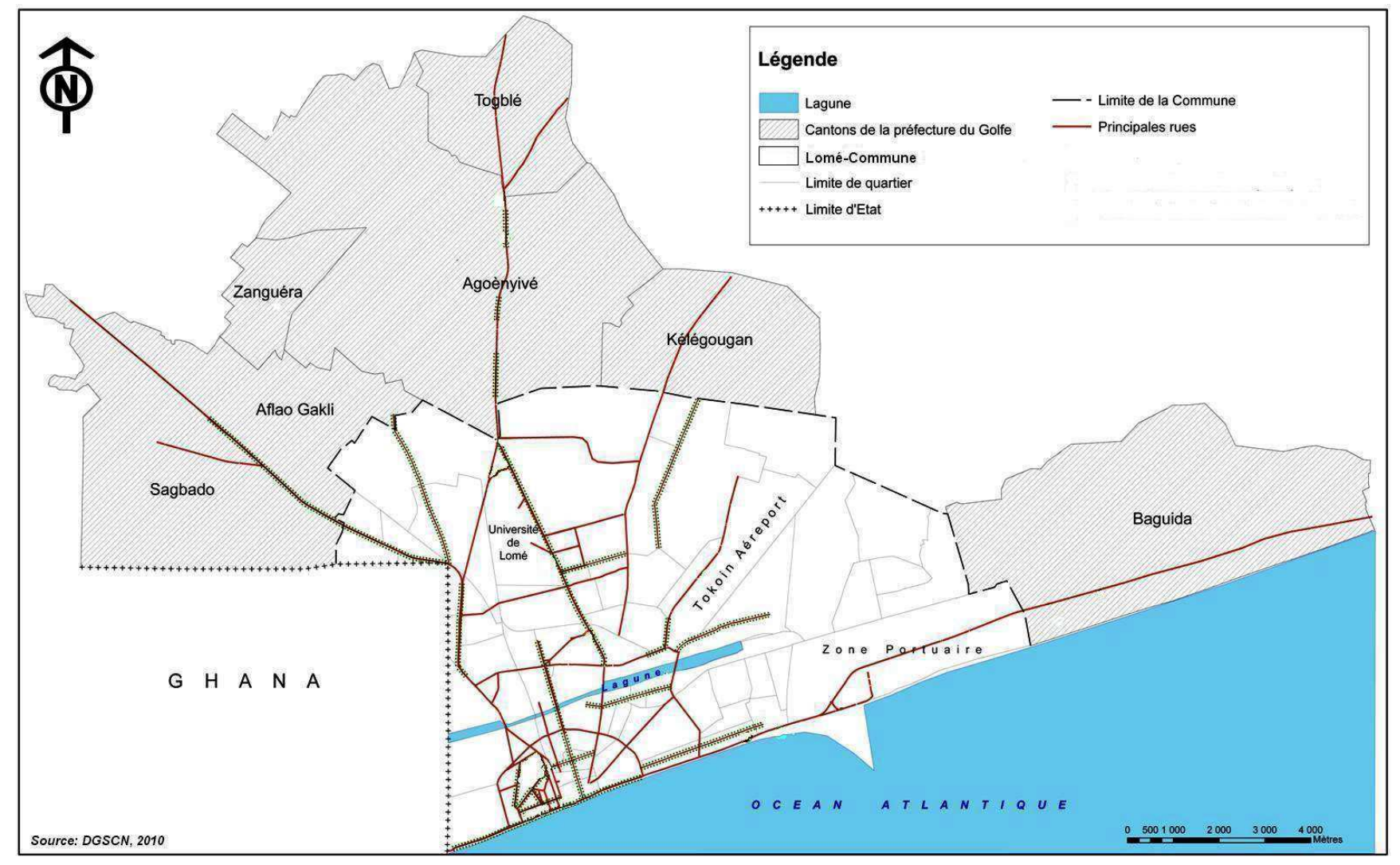

Figure 1 : Localisation de la zone d'étude. 
Suivant le décret $\mathrm{n}^{\circ} 011-2006 / \mathrm{PR}$ du 8 février 2006 portant organisation, fonctionnement et attributions du ministère de la ville et de l'urbanisme, la gestion des espaces verts de la ville incombe à la DEVMHE et aux collectivités territoriales. Ce décret stipule que la DEVMHE a pour mission de définir et de mettre en application les orientations en matière d'aménagement d'espaces verts, de protection des monuments historiques et d'embellissement du milieu urbain.

Par ailleurs, la loi n²007-011 du 13 mars 2007 portant décentralisation et liberté locale établit la mission de l'atelier espaces verts de la direction des services techniques de la municipalité de Lomé et du service technique de la préfecture du Golfe en matière de espaces verts. En effet, l'article 53 précise que dans le domaine de la gestion des ressources naturelles et protection de l'environnement, les collectivités territoriales sont chargées de la création et entretien des espaces verts. La mission de chaque collectivité relève de son ressort territorial.

\section{Allocations budgétaires et capacités en ressources humaines pour les aménagements en espaces verts}

Conformément au cadre institutionnel, la conception et l'aménagement des espaces verts incombent à la DEVMHE. La phase d'exploitation (la gestion) est dévolue à la municipalité de Lomé et au service technique de la préfecture du Golfe en fonction du ressort territorial (périmètre communal pour la municipalité et préfecture du Golfe pour le service technique).

Cependant, l'une des faiblesses majeures de ces services chargés de la gestion des espaces verts est l'insuffisance des moyens financiers et humains (Tableaux 2 et 3) pour mieux gérer les espaces verts du point de vue de leur conservation, protection et valorisation.

La DEVMHE compte 9 agents dont 7 cadres de conception et 2 agents d'exécution. Son budget d'investissement, faible, fluctue d'année en année. Depuis son opérationnalisation en 2009, la direction a obtenu en 2010 un budget de 35 millions de F CFA pour élaborer un document de planification des espaces verts dans la ville de Lomé (Stratégie en matière des espaces verts et des monuments dans la ville de Lomé). Cette stratégie validée en 2010 peine à connaître une mise en œuvre diligente et efficace. Deux années plus tard, un budget de 360 millions lui a été alloué pour entreprendre des travaux d'aménagement en espace vert autour du bassin de rétention d'eaux pluviales du quartier Agbalépédogan. La direction ne dispose d'aucun matériel de réalisation de ces activités.

L'atelier espaces verts de la municipalité est composé d'un aménagiste et des agents d'appui contractuel. Son budget annuel et constant de 17604000 de F CFA est destiné à l'entretien de l'espace vert de la place de l'indépendance, à l'élagage des arbres de la ville, à la taille ou coupe des arbustes et des haies et au sarclage des herbes sur les principales artères bordées d'arbres en alignement et sur les terre-pleins centraux fleuris. Le manque de personnel qualifié dans ce service semble suppléer par des contrats de travail signés avec des sociétés prestataires de la place. Le matériel et les équipements de travail de l'atelier se résument en quelque matériel de petits jardinages.

Le budget total du service technique de la préfecture du Golfe destiné à l'entretien (taille ou coupe) des arbustes et des haies et au sarclage des herbes sur les principales artères bordées d'arbres en alignement et sur les terre-pleins centraux fleuris sur la période 2010-2015 est de 19 millions F CFA. 
Fluctuant d'année en année, ce budget a permis de réaliser des travaux en régie contrôlée en 2010 et 2011. Depuis 2012, les travaux sont réalisés par lettre de commande comme précédemment cité. Un aménagiste et 03 techniciens (personnel d'appui) constituent l'équipe espace vert du service.

Textes juridique et réglementaire de la politique publique en matière des espaces verts

Au Togo, deux principaux textes règlementent la politique publique en matière des espaces verts. Il s'agit de la Constitution de la $4^{\text {ème }}$ République et la loi n²008-005 du 30 mai 2008 portant loi-cadre sur l'environnement.

En effet, la Constitution de la $4^{\text {ème }}$ République du 14 octobre 1992 dispose en son article 41 que toute personne a droit à un environnement sain et que l'État veille à la protection de cet environnement. L'article 84 détermine le domaine de compétence de la loi, et mentionne que la loi fixe les règles concernant la protection et la promotion de l'Environnement et la conservation des ressources naturelles. L'aménagement des espaces verts concourt à la mise en œuvre de la politique de protection et de gestion de l'environnement. Il rentre donc dans le cadre des politiques et stratégies de développement durable du pays.

La loi n²008-005 du 30 mai 2008 portant loi-cadre sur l'environnement garantit un cadre de vie agréable aux populations des agglomérations urbaines, périurbaines et rurales. Elle fait de l'aménagement des espaces verts, un outil de gestion de l'environnement et un gage de développement durable. La gestion de ces espaces verts est régie par plusieurs dispositions dont certaines répondent à l'orientation et à l'engagement que l'Etat doit prendre au regard des différents espaces aménagés. L'article 92 stipule que l'État veille à la protection des agglomérations urbaines et rurales, des infrastructures et équipements, en vue de garantir un cadre de vie agréable aux populations. L'article 94 précise que les agglomérations urbaines doivent comporter des terrains à usage récréatif et des zones d'espaces verts selon une proportion harmonieuse fixée par les règlements d'urbanisme et la législation forestière, compte tenu, notamment des superficies disponibles, du coefficient d'occupation $\mathrm{du}$ sol et de la population résidentielle. L'article 96 dispose que les embellissements qui font l'objet d'aménagements paysagers regroupent les espaces verts, les plantations d'alignement, les jardins, les ceintures vertes, les parterres, les parcs urbains, les squares, les embellissements des sites et des voies publiques. L'article 97 précise par contre que les aménagements paysagers peuvent être entrepris par des personnes physiques ou morales, de droit public ou privé, sur autorisation des autorités locales alors que l'article 98 fixe les normes d'aménagement en précisant que les projets d'aménagements paysagers d'intérêt public doivent être soumis à l'examen des services techniques compétents et leur exécution doit faire l'objet de contrôles réguliers. 
B. POLORIGNI et al. / Int. J. Biol. Chem. Sci. 9(4): 1888-1901, 2015
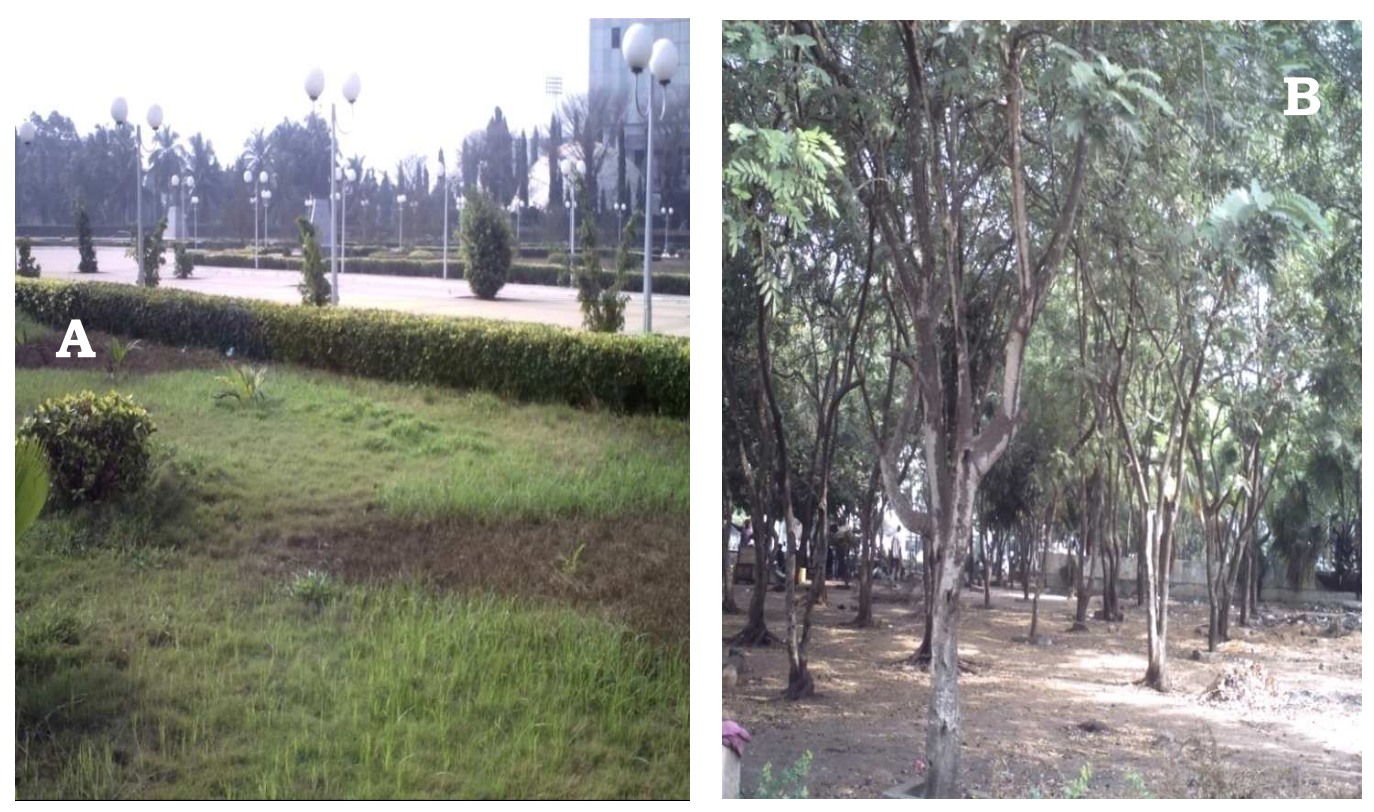

Photo 1: Espaces verts. A: Place de l'indépendance ; B: Place publique Maman N'Danida.

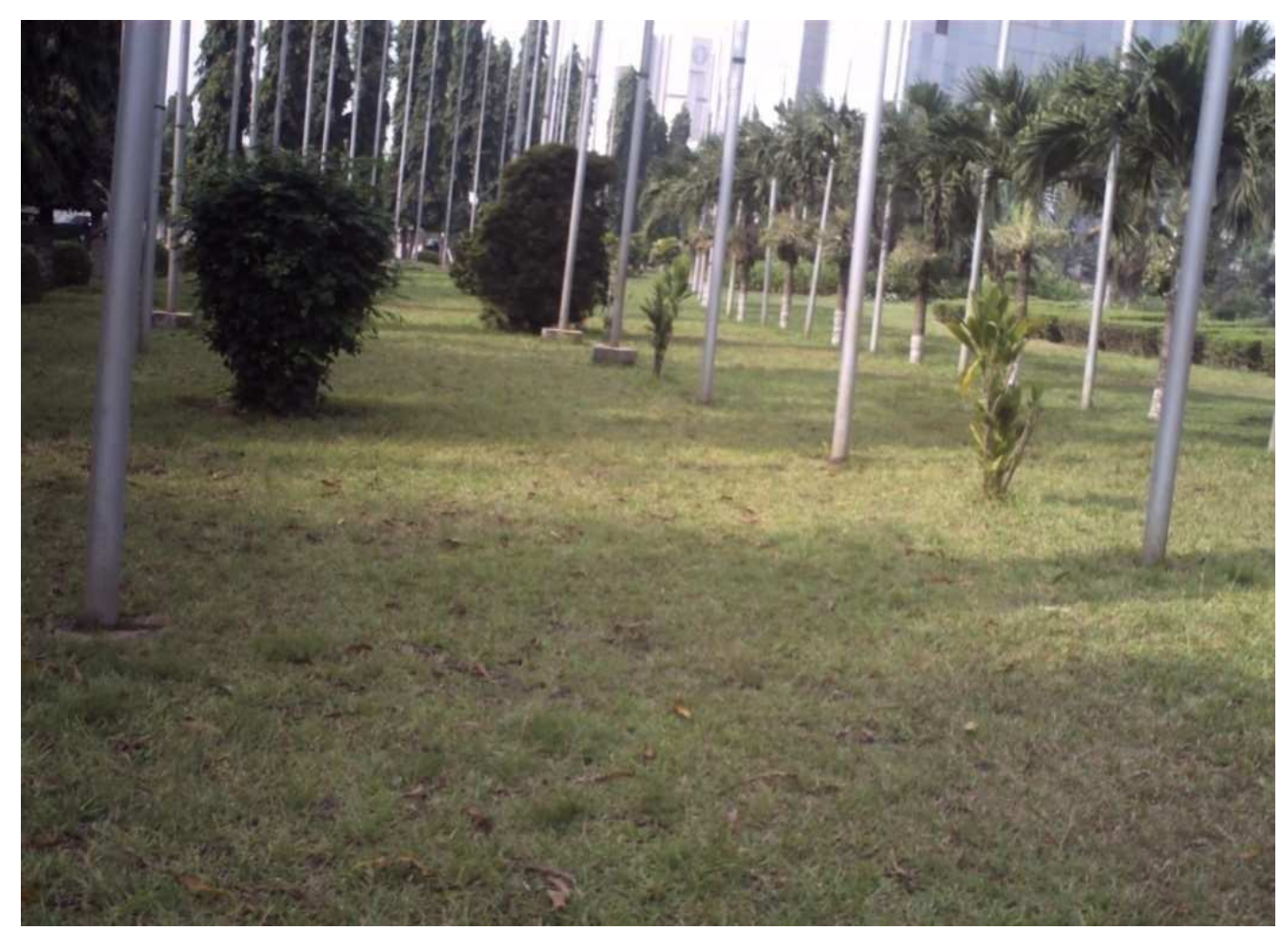

Photo 2 : Jardin privé de l'hôtel 02 février. 
Tableau 1 : Types d'espaces verts étudiés dans la ville de Lomé.

\begin{tabular}{|c|c|c|c|c|c|}
\hline \multirow[b]{2}{*}{ Type d'Espaces } & \multirow{2}{*}{$\begin{array}{c}\text { Nombre d'espace } \\
\text { identifié/linéaire de } \\
\text { voies bordées d'arbres } \\
\text { plantés en alignement }\end{array}$} & \multirow[b]{2}{*}{$\begin{array}{l}\text { Superficie } \\
\qquad\left(\mathbf{m}^{2}\right)\end{array}$} & \multicolumn{2}{|c|}{ Etat de l'aménagement } & \multirow[b]{2}{*}{ Espèces dominantes } \\
\hline & & & $\begin{array}{c}\text { Nombre/ } \\
\text { linéaire de } \\
\text { voie Aménagé }\end{array}$ & $\begin{array}{c}\text { Nombre/ linéaire } \\
\text { de voie non } \\
\text { Aménagé }\end{array}$ & \\
\hline $\begin{array}{l}\text { Artères bordées } \\
\text { d'arbres en } \\
\text { alignement }\end{array}$ & $176 \mathrm{~km}$ & 174000 & $58 \mathrm{~km}$ & $118 \mathrm{~km}$ & $\begin{array}{l}\text { Khaya senegalensis, Azadirachta } \\
\text { indica, Ficus spp. }\end{array}$ \\
\hline Jardins publics & 15 & 62047 & 6 & 9 & $\begin{array}{c}\text { Azadirachta indica, Zoysia } \\
\text { tenuifolia, Khaya senegalensis, } \\
\text { Ficus retusa, Cassia siamea, Elaeis } \\
\text { guineensis }\end{array}$ \\
\hline Jardins privés & 16 & 454181 & 16 & 0 & $\begin{array}{c}\text { Zoysia tenuifolia, Ficus retusa, } \\
\text { Ixora macrothyrsa }\end{array}$ \\
\hline $\begin{array}{l}\text { Aires de jeux } \\
\text { engazonnés }\end{array}$ & 3 & 418600 & 3 & - & $\begin{array}{c}\text { Azadirachta indica, Zoysia } \\
\text { tenuifolia, Ficus retusa }\end{array}$ \\
\hline TOTAL & - & 1108828 & - & - & \\
\hline
\end{tabular}


Tableau 2 : Evolution des budgets d'investissement des trois principaux acteurs de la gestion des espaces verts.

\begin{tabular}{lccc}
\hline & \multicolumn{2}{c}{ Budgets d'investissement en matière d'espaces verts (en F CFA) } \\
\cline { 2 - 4 } Années & $\begin{array}{c}\text { Direction des Espaces Verts, des } \\
\text { Monuments Historiques et de } \\
\text { l'Embellissement }\end{array}$ & $\begin{array}{c}\text { Atelier espaces verts de la } \\
\text { direction des services } \\
\text { techniques de la municipalité } \\
\text { de Lomé }\end{array}$ & $\begin{array}{c}\text { Service technique de la } \\
\text { préfecture du Golfe }\end{array}$ \\
\hline 2010 & 35000000 & 17604000 & 500000 \\
2011 & 0 & 17604000 & 1500000 \\
2012 & 0 & 17604000 & 3000000 \\
2013 & 360000000 & 17604000 & 4000000 \\
2014 & 0 & 17604000 & 5000000 \\
2015 & 0 & 17604000 & 5000000 \\
Total & $\mathbf{3 9 5 0 0 0 0 0 0}$ & $\mathbf{1 0 5 6 2 4 0 0 0}$ & $\mathbf{1 9 0 0 0 0 0 0}$ \\
\hline
\end{tabular}


Tableau 3 : Effectif du personnel technique des trois principaux acteurs de la gestion des espaces verts.

\begin{tabular}{lccc}
\hline Profil du personnel & $\begin{array}{c}\text { Direction des Espaces } \\
\text { Verts, des Monuments } \\
\text { Historiques et de } \\
\text { l'Embellissement }\end{array}$ & $\begin{array}{c}\text { Atelier espaces verts } \\
\text { de la municipalité de } \\
\text { Lomé }\end{array}$ & $\begin{array}{c}\text { Service technique de la } \\
\text { préfecture du Golfe }\end{array}$ \\
\hline Botaniste & - & - & - \\
Paysagiste & - & - & - \\
Ingénieur phytosanitaire & 01 & 01 & - \\
Aménagiste & - & - & - \\
Architecte & - & - & - \\
Urbaniste & - & - & - \\
Géographe & 05 & - & - \\
Economiste & - & - & - \\
Génie civil & - & - & - \\
Sociologue & 01 & 13 & $\mathbf{4}$ \\
Personnel d'appui & 02 & $\mathbf{1 4}$ & - \\
Total & $\mathbf{0 9}$ & & \\
\hline
\end{tabular}




\section{DISCUSSION}

La présente étude révèle qu'à Lomé, le nombre des espaces verts est très insuffisant et varie selon les quartiers. Ceux-ci représentent $0,4 \%$ de la superficie totale de la ville. La métropole dispose de moins de $1 \mathrm{~m}^{2}$ d'espaces verts par habitant en moyenne, inférieur aux $10 \mathrm{~m}^{2}$ recommandés par l'OMS et très loin des $20 \mathrm{~m}^{2}$ dont disposent les villes de Paris et de New York. Comparée à d'autres villes moins grandes et moins importantes que Paris, cette superficie par habitant d'espaces verts reste toujours moins importante à Lomé qu'à Casablanca $\left(2 \mathrm{~m}^{2}\right)$, à Bagneux $\left(4,79 \mathrm{~m}^{2}\right)$, en Californie $\left(5 \mathrm{~m}^{2}\right)$ ou à Issy-les-Moulineaux (7 $\mathrm{m}^{2}$ ) (Actevi, 2010 ; Izddine, 2010).

Il est important de signaler que la présente étude a porté sur les espaces verts d'utilité publique et des institutions privées. Elle n'a pas pris en compte les espaces verts des résidences privées où il est possible de trouver pour les habitants aisés ou résidants dans les logements des responsables des institutions financières ou de l'Etat, des espaces verts de plus de $2400 \mathrm{~m}^{2}$ pour une concession de $4800 \mathrm{~m}^{2}$ abritant environ 5 habitants. Dans ces cas, le ratio espaces verts par habitant est nettement supérieur aux $10 \mathrm{~m}^{2}$ recommandés par l'OMS.

Malgré cette insuffisance, la priorité est aujourd'hui accordée à la construction des infrastructures routières, des bureaux et immeubles de standing. Sur les plans administratifs et cadastraux, les réserves administratives et placettes allouées aux éventuels aménagements paysagers sont squattés par les populations soit pour des marchés commerciaux de fortune ou pour des habitations.

L'absence de vision urbanistique de la ville (Kassay, 2010) explique cette situation alors que seule la volonté de favoriser les promotions immobilières a été prise en compte. Les arbres centenaires des artères arborés et les principaux parcs et jardins de la ville sont détruits (exemple des Khayas du Collège Saint Joseph, du jardin de l'Aéroport international Gnassingbe Eyadema) ou très mal entretenus (Parc Anani Santos, jardin de l'hôtel 2 février). C'est dans ce contexte que la perte de la biodiversité est dorénavant considérée comme un des enjeux environnementaux les plus présents concernant la végétation urbaine (McPherson, 2006). Les caractéristiques physicochimiques de la ville, différentes de celles des milieux naturels, (température moyenne plus élevée, altération du régime hydrique, alternance des jours et des nuits perturbée par les éclairages publics, fragmentation des habitats, etc.) rendent le développement d'un arbre plus ardu et peuvent contribuer à la perte de certaines espèces qui ne sont plus en mesure de s'adapter à ces nouvelles conditions. Le processus d'homogénéisation biotique est ainsi observé dans les écosystèmes urbains. Ces derniers, modifiés par de nouveaux aménagements, offrent alors des opportunités de colonisation pour les espèces exogènes; ce qui a pour conséquence la diminution de la variation des habitats ainsi qu'un appauvrissement de la diversité d'espèces natives (Arce, 2009).

Le cadre institutionnel en vigueur est peu favorable à la concertation et à l'implication des populations dans l'élaboration des politiques de gestion des espaces aménagés. En effet, la gestion des espaces verts est émiettée dans les attributions des trois principaux acteurs que sont la DEVMHE du ministère de l'urbanisme et de l'habitat, l'atelier espaces verts de la direction des services techniques de la municipalité et le service technique de la préfecture du Golfe. Si les aménagements en espaces verts peuvent émaner de tout projet dans leur phase de conception, la phase de réalisation nécessite l'intervention de la DEVMHE et celle d'exploitation est du ressort de l'atelier espace vert de la municipalité et du service technique de la préfecture. Mais, dans la réalité, ces différentes phases sont souvent mal définies et chaque service intervient dans l'exécution des trois phases, sans concertation des autres services et donc sans harmonisation (Kombaté, 2004).

Les ressources financières pour les aménagements en espaces verts sont insuffisantes. Les trois services totalisent un budget de 519624000 millions de F CFA sur 6 ans. Ce montant est très faible pour une valorisation des aménagements en espaces verts. Le budget constant de l'atelier espace vert de la municipalité ne permet pas de créer 
de nouveaux espaces verts car il est prioritairement destiné aux entretiens de deux aménagements en espaces verts. Il en est de même pour le budget du service technique de la préfecture du Golfe. La DEVMHE quant à elle, n'a pu aménager qu'un seul site en 6 ans avec son budget. Au regard de ces ressources financières insuffisantes, comment pourrait-on atteindre la proportion de $10 \mathrm{~m}^{2} \mathrm{~d}$ 'espace vert par habitant sachant que le taux annuel d'accroissement démographique de la ville de Lomé est 5\% (DGSCN, 2010) ?

Pour l'ensemble des services concernés, il se pose aussi le problème de qualification du personnel. En effet, aucun paysagiste ni botaniste ne figure parmi le personnel des services techniques en charge des aménagements paysagers à Lomé. Les forestiers et planificateurs professionnels jouent encore un rôle mineur dans les initiatives d'écologisation car ils sont rarement consultés (N'Zala, 2002). De plus, aucun programme de sensibilisation en matière d'éducation environnementale n'est mené par ces services face au manque de civisme d'une couche de la population qui se livre à des actes répétés de vandalisme sur les espaces verts qui sont transformés en marchés pirates (Kassay, 2010), en dépotoirs et lieux d'aisance.

A ces contraintes administratives et institutionnelles s'ajoute la faible incidence de la loi n²008-005 du 30 mai 2008 portant loicadre sur l'environnement au Togo. Les articles 94 et 98 de cette loi précisent respectivement les normes et conditions dans lesquelles tout aménagement paysager doit se réaliser. Mais dans la pratique, ces dispositions ne sont nullement observées. Plusieurs projets d'aménagements paysagers sont exécutés par des privés ou acteurs publics sans consultation des services techniques en charge de la gestion. Par ailleurs, le chapitre 3 de cette loi énonce les sanctions liées aux infractions tout en n'étant pas spécifiques aux aménagements en espaces verts. Les peines restent relatives aux activités susceptibles de porter atteinte à la faune et à la flore ; ce qui ne facilite pas alors l'application des sanctions concernant les atteintes aux aménagements en espaces verts et rend la loi $\mathrm{n}^{\circ} 2008-005$ superficielle.
Au-delà de cette loi générale, la réglementation en matière d'assainissement, d'hygiène et des espaces verts en particulier doit relever des collectivités territoriales. Mais il se trouve qu'aucune réglementation communale n'existe en matière des espaces verts. Elle se limite à l'inscription sur des panneaux «Interdit de jeter des ordures ménagères. Amende 5000 F CFA». Cette faible implication des collectivités territoriales peut s'expliquer par la non application de la loi n²007-011 du 13 mars 2007 relative à la décentralisation et aux libertés locales.

\section{Conclusion}

Cette étude a permis d'évaluer la politique de gestion des espaces verts de la ville de Lomé par les autorités publiques. L'état des lieux indique que les espaces verts de la ville sont constitués de jardins publics et privés, d'aires de jeux engazonnés et d'artères bordées d'arbres en alignement. La superficie totale des espaces verts est de $1108828 \mathrm{~m}^{2} \mathrm{et}$ la proportion d'espace vert par habitant est de $0,75 \mathrm{~m}^{2}$. Ce qui atteste d'un déficit en espace vert car cette valeur est de loin inférieure à la norme de l'OMS. Trois services sont chargés des aménagements en espaces verts et la loi n 2008 -005 du 30 mai 2008 portant loi-cadre sur l'environnement réglemente ces aménagements. Cependant, ce cadre institutionnel et juridique est caractérisé par des dysfonctionnements et manquements. Les ressources financières et humaines restent insuffisantes pour leur fonctionnement.

Pour lever les contraintes identifiées, il importe de moderniser les services techniques en charge de la gestion des espaces verts, d'accroître leur budget d'investissement, d'instaurer un cadre de concertation et coordination des actions, de former le personnel des services techniques, de mener des campagnes de sensibilisation en matière d'éducation environnementale, d'actualiser les textes régissant les espaces verts et d'appliquer des sanctions et amendes à l'encontre des contrevenants.

La ville de Lomé est caractérisée par une extension spatiale sans cesse croissante se traduisant par une expansion des aires urbaines. Le principal défi sera d'inscrire les aménagements en espaces verts dans le cadre 
de son développement durable avec une gestion participative de tous les acteurs de ce développement.

\section{REFERENCES}

Actevi. 2010. Livre blanc, les espaces verts à Issy. Action citoyenne pour les transports et l'environnement de la Ville d'Issy-les-Moulineaux.

http://www.actevi.fr/lists/documents/livr e_blanc_actevi_espaces_verts_a_issy.pd $\mathrm{f}$

Adjaho D. 2010. Développement des espaces géographiques : exemple $\mathrm{du}$ terroir d'Assomé dans la basse vallée du Zio. Mémoire de maîtrise en géographie, Université de Lomé, Lomé, 164 p.

Afidégnon D. 1999. Les mangroves et les formations associées du sud-est du Togo: analyse écofloristique et cartographique par télédétection spatiale. Thèse de doctorat, Université de Lomé, Lomé, 237 p.

Akpagana K, Bouwessidjaou E, Edorh T, Guyot M, Roussel B. 1993. L'Ecosystème des mangroves et les formations affiliées du sud Togo. Diop S. (ed). Conservation et Utilisation Rationnelle des Forêts de Mangroves de l'Amérique Latine et de l'Afrique, 2, ITTO/ISME-UNESCO/COMARAF :

Dakar ; 108-112.

Ali Idrissou YK. 2009. Quelle place pour les espaces verts dans le développement de la ville de Porto-Novo au Bénin? Aménagement d'un parc urbain sur la dépression de Zounvi. Mémoire de fin d'études à l'EAMAU, Lomé, 152p.

Ali-Khodja A. 2010. Aménagement urbain : la problématique de l'espace vert public dans la ville de Constantine. Sciences et Technologie, 32: 9-18.

Arce S. 2009. Conservation de la biodiversité en milieu urbain : considérations écologiques et analyse du cadre de Montréal. Mémoire de maîtrise en environnement, Université du Québec à Montréal, Montréal, Québec, 150 p.

Arnberger A, Renate E. 2012. The influence of green space on community attachment of urban and suburban residents. Urban Forestry \& Urban Greening, 11: 41-49.
Batawila K. 1997. Recherches botaniques sur les formations dégradées et jachères de la plaine côtière du sud-Togo. Mémoire de DEA, biologie végétale, Université de Lomé, Lomé, 65p.

CC-PNAE (Cellule de Coordination du Plan National d'Action pour l'Environnement) 2003. Stratégie de Conservation et l'Utilisation Durable de la Diversité Biologique. Ministère de l'Environnement et des Ressources Forestières, Togo, 164 p.

Choumert J. 2009. Analyse économique d'un bien public local: les espaces verts. Thèse de doctorat, Université d'Angers, $425 \mathrm{p}$.

Dakissaga W. 2006. Requalification des espaces verts en espaces qualificatifs de loisir : un pôle ludique sur les berges des lacs de Ouagadougou. Mémoire de fin d'étude, EAMAU, Lomé, 192 p.

Davies ZG, Edmonson JL, Heinemeyer A, Leake JR, Gaston KJ. 2011. Mapping an urban ecosystem service: quantifying above-ground carbon storage at a citywide scale. Journal of Applied Ecology, 48: 1125-1134.

Direction de la météorologie nationale. 2012. Evolution de la température et des précipitations de la ville de Lomé de 2000 à 2012, 3 p.

DGSCN (Direction Générale de la Statistique et de la Comptabilité Nationale), 2010. Résultats définitifs du recensement général de la population et de l'habitat. Ministère auprès du Président de la République chargé de la planification, du développement et de l'aménagement du territoire, Direction générale de la statistique et de la comptabilité nationale, Togo, $65 \mathrm{p}$.

ELOM OP. 2011. La privatisation des espaces publics dans la ville de Yaoundé (Cameroun) : le cas des activités commerciales. Afrique et Développement, 36(1) : 1-11.

Guezere A. 2008. "Oléyia" (taxi moto): acteurs et usagers d'un mode de transport artisanal récent à Lomé. Thèse de Doctorat d'Etat, Université de Lomé, Lomé, 458 p. 
Hoshino T, Kuriyama K. 2010. Measuring the benefits of neighbourhood park amenities: Application and comparison of spatial hedonic approaches. Environmental et Resource Economics, 45(3) : 429-444.

Institut d'Aménagement et d'Urbanisme, 2009. La Desserte en Espaces Verts, un Outil de Suivi de la Trame Verte d'Agglomération : Île de France, Paris, juin 2009, 16 p.

Izddine M. 2010. Casablanca a soif de vert. L'observateur.

http://www.maghress.com/fr/lobservateu $\mathrm{r} / 2342$

Kassay NJ. 2010. La politique publique de la gestion des espaces verts par l'hôtel de ville de Kinshasa. $12^{\text {ème }}$ Assemblée générale du CODESRIA, Administrer l'espace public africain, du 07 au 11 décembre, 13-46.

Kokou K. 1998. Les mosaïques forestières au sud du Togo : biodiversité, dynamique et activités humaines. Thèse de doctorat de l'Université de Montpellier II, France, $140 \mathrm{p}$.

Kombaté A. 2004. Problématique de la gestion des espaces verts au Togo : Cas de la ville de Lomé. Mémoire de fin de formation. Institut National de Formation Agricole (INFA) de Tové, Togo, $40 \mathrm{p}$.

Kubal C, Haase D, Meyer V, Scheuer S. 2009. Integrated urban flood risk assessmentAdapting a multicriteria approach to a city. Natural Hazards and Earth System Science, 9(6): 1881-1895.

Loughner CP, Allen DJ, Zhang D-L, Pickering KE, Dickerson R, Landry L. 2012. Roles of urban tree canopy and buildings in urban heat island effects: parameterization and preliminary results. Journal of Applied Meteorology and Climatology, 51(10): 1775-1793.

McPherson EG. 2006. Getting more than we pay for. City Trees, 201(7): 9-12.
Mehdi L, Di pietro F. 2009. L'espace vert public, lieu d'interactions entre société et biodiversité. Projet de paysage, http://www.projetsdepaysage.fr. 17p.

NassaDabie A. 2009. Crise de la nature dans l'agglomération abidjanaise : l'exemple de la colonisation des espaces verts par l'habitat et les commerces dans la commune de Cocody. L'archive halvous, http://www.hal.archivesouvertes.fr, $10 \mathrm{p}$.

N'Zala D. 2002. L'arbre en ville, étude de foresterie urbaine à Brazzaville, Congo. Bois et Forêts des Tropiques, 272(2): 88-92.

Polorigni B. 2012. Les espaces verts dans les villes africaines, diagnostic et perspectives : cas de la ville de Lomé. Mémoire de DEA, gestion de l'environnement, Université de Lomé, Lomé, 104 p.

Richardson, Elizabeth A, Mitchell R. 2010.Gender differences in relationships between urban green space and health in the United Kingdom. Social Science and Medicine, 17(3): 568-575.

Säumel I, Kowarik I, Butenschön S. 2010.Green traces from past to future: the interplay of culture and ecological processes in European historical parks. Acta Horticulturae, 881: 993-938.

Taylor, Andrea F, Kuo E. 2009.Children with attention deficits concentrate better after walk in the park. Journal of Attention Disorders, 12(5): 402-409.

Van Dillen, Sonja M, Sjerp de Vries, Peter P, Groenewegen, Spreeuwenberg 2012. Greenspace in urban neighbourhoods and residents' health: adding quality to quantity. Journal of Epidemiology and Community Health, 66(6): 353-376. 